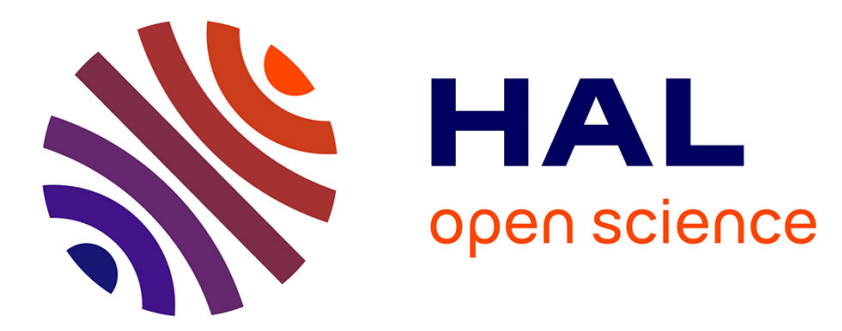

\title{
Dystrophin Dp71 is Critical for Stability of the DAPs in the Nucleus of PC12 Cells
}

Marcela Villarreal-Silva, Rocío Suárez-Sánchez, Rafael Rodríguez-Muñoz, Dominique Mornet, Bulmaro Cisneros

\section{- To cite this version:}

Marcela Villarreal-Silva, Rocío Suárez-Sánchez, Rafael Rodríguez-Muñoz, Dominique Mornet, Bulmaro Cisneros. Dystrophin Dp71 is Critical for Stability of the DAPs in the Nucleus of PC12 Cells. Neurochemical Research, 2010, 35 (3), pp.366-373. 10.1007/s11064-009-0064-z . hal-02547025

\section{HAL Id: hal-02547025 \\ https://hal.umontpellier.fr/hal-02547025}

Submitted on 19 Apr 2020

HAL is a multi-disciplinary open access archive for the deposit and dissemination of scientific research documents, whether they are published or not. The documents may come from teaching and research institutions in France or abroad, or from public or private research centers.
L'archive ouverte pluridisciplinaire HAL, est destinée au dépôt et à la diffusion de documents scientifiques de niveau recherche, publiés ou non, émanant des établissements d'enseignement et de recherche français ou étrangers, des laboratoires publics ou privés. 


\title{
Dystrophin Dp71 is Critical for Stability of the DAPs in the Nucleus of PC12 Cells
}

\author{
Marcela Villarreal-Silva • Rocío Suárez-Sánchez • \\ Rafael Rodríguez-Muñoz $\cdot$ Dominique Mornet • \\ Bulmaro Cisneros
}

\begin{abstract}
We have adopted the PC12 cell line as in vitro cell model for studying Dp71 function in neuronal cells. These cells express a cytoplasmic (Dp71f) and a nuclear (Dp71d) isoform of Dp71 as well as various dystrophinassociated proteins (DAPs). In this study, we revealed by confocal microscopy analysis and Western blotting evaluation of cell fractions the presence of different DAPs ( $\beta$-dystroglycan, $\beta$-dystrobrevin, $\varepsilon$-sarcoglycan and $\gamma 1$ syntrophin) in the nucleus of PC12 cells. Furthermore, we established by immunoprecipitation assays that Dp71d and the DAPs form a dystrophin-associated protein complex (DAPC) in the nucleus. Interestingly, depletion of Dp71 by antisense treatment (antisense-Dp71 cells) provoked a drastic reduction of nuclear DAPs, which indicates that Dp71d is critical for DAPs stability within the nucleus. Although Up71, the utrophin gene product homologous to Dp71, exhibited increased expression in the antisenseDp71 cells, its scarce nuclear levels makes unlikely that could compensate for Dp71 nuclear deficiency.
\end{abstract}

\footnotetext{
M. Villarreal-Silva $\cdot$ R. Suárez-Sánchez · R. Rodríguez-Muñoz $\cdot$ B. Cisneros $(\bowtie)$

Departamento de Genética y Biología Molecular, Centro de Investigación y de Estudios Avanzados del IPN, Avenida Instituto Politécnico Nacional 2508, Apartado Postal 14-740, CP 07360 Mexico, DF, Mexico

e-mail: bcisnero@cinvestav.mx

D. Mornet

Département de Physiologie des Interactions (EA701), Institut de Biologie, Bvd Henry IV, 34062 Montpellier, France
}

Keywords Dystrophin Dp71 · Utrophin · DAPs · Nucleus · Nuclear matrix · Protein stability

\section{Introduction}

Duchenne muscular dystrophy (DMD) is a lethal X-chromosome-linked hereditary disorder characterized by progressive degeneration of muscle tissue [1]. The disease is caused by mutations in the DMD gene that disrupt the function of dystrophin, the main DMD gene product in muscle tissue [2]. Dystrophin is a $427 \mathrm{kDa}$ cytoskeletal protein that interacts with transmembrane (dystroglycans and sarcoglycans) and cytoplasmic (syntrophins, dystrobrevins nNOS) proteins, known collectively as dystrophinassociated proteins (DAPs). The protein complex formed by dystrophin and DAPs, which is called dystrophinassociated protein complex (DAPC), is involved in sarcolemmal integrity and signal transduction $[3,4]$.

DMD gene exhibits complex regulation, it produces different full-length and N-terminal-truncated dystrophin isoforms, which are termed according with their respective molecular weights as Dp427, Dp260, Dp140, Dp116 and Dp71 [5-8]. Dp71 is the most abundant DMD gene product in the nervous system, where it associates with the DAPs to assemble a DAPC-like [9-11]. Although the function of Dp71 in the nervous system remains to be elucidated, indirect experimental evidence suggests that this protein has an important role therein. It has been reported that C-terminal mutations in the DMD gene that disrupt the expression of Dp71 are linked with cognitive impairment of DMD patients [12, 13]. Furthermore, expression of Dp71 is induced during development of the nervous system $[14,15]$. 
With the aim of finding a role for Dp71 in neuronal cells, we have started a long term study in the PC12 cells, a cell line broadly used for differentiation studies [16, 17]. We have previously established that PC12 cells express two different splicing isoforms of Dp71, which display specific subcellular distribution each one from each other: Dp71d has a predominant nuclear localization, while Dp71f is distributed solely to the cytoplasm [18]. In our previous study, we revealed that association and distribution of Dp71d to the nuclear matrix are modulated during the NGF-induced neuronal differentiation of PC12 cells, which indicates that Dp71d is a component of the nuclear matrix that might participate in the nuclear modeling taking place during this process [19].

In this study, we revealed that besides Dp71d, several DAPs $(\beta$-dystroglycan, $\beta$-dystrobrevin, $\varepsilon$-sarcoglycan and $\gamma 1$-syntrophin) are also present in the nucleus of PC12 cells. Furthermore, we found that Dp71d associates with these DAPs to assemble a nuclear DAPC. Interestingly, depletion of Dp71 by an antisense treatment provoked a marked reduction of the DAPs in the nucleus of PC12 cells, which indicates that Dp71d is crucial for stability of the nuclear DAPs.

\section{Experimental Procedure}

\section{Cell Culture}

PC12 cells were cultured in RPMI-1640 (Invitrogen, Carlsbab, CA, USA), supplemented with $5 \%$ fetal bovine serum (FBS), $10 \%$ horse serum (inactivated at $56^{\circ} \mathrm{C}$ for $30 \mathrm{~min}$ ), $100 \mathrm{U} / \mathrm{ml}$ penicillin and $100 \mu \mathrm{g} / \mathrm{ml}$ streptomycin, and maintained at $37^{\circ} \mathrm{C}$ in a humidified atmosphere containing 5\% CO2. PC12 derivative clones (antisense-Dp71 cells) and control vector were obtained by stable transfection of PC12 cells with plasmids pDp71As and p $\Delta$ GFP respectively, as described previously [17].

\section{Antibodies}

Polyclonal antibodies against $\beta$-dystroglycan (LG5), $\alpha$-sarcoglycan (Sarco 3), $\beta$-sarcoglycan (G4), $\delta$-sarcoglycan (Nini), $\alpha 1$-syntrophin (C4) [20], utrophin (CUT) [21, 22], $\gamma 1$-syntrophin (LG1) and $\varepsilon$-sarcoglycan (LG7) were kindly provided by Dr. Dominique Mornet. Antibodies directed to $\alpha 1$-, $\alpha 2$ - and $\beta$-dystrobrevin ( $\beta$ CT-FP) [23] and Dp71 (2166) [24] were provided by Dr. Dereck Blake. Antibody antiactin was supplied by Dr. Manuel Hernández [25]. The polyclonal antibodies against Dp71 (DPC20, used for the immunoprecipitation assays) and $\alpha 1$-syntrophin (N-19, used for Western blotting assays) were purchased from Santa Cruz Biotechnology, Inc. (Santa Cruz, CA, USA).
Immunofluorescence and Confocal Microscopy Analysis

Cells, cultured on coverslips for $24 \mathrm{~h}$, were washed with PBS, fixed with $4 \%$ paraformaldehyde in PBS for $10 \mathrm{~min}$ at room temperature, permeabilized with $0.2 \%$ triton $\mathrm{X}-100$ in PBS for $5 \mathrm{~min}$ at $4{ }^{\circ} \mathrm{C}$, blocked with $0.5 \%$ gelatin and $1.5 \%$ FBS in PBS for $20 \mathrm{~min}$ at room temperature and incubated overnight at $4{ }^{\circ} \mathrm{C}$ with the corresponding primary antibodies. Primary antibodies were detected by incubating slides for $1 \mathrm{~h}$ with a secondary anti-rabbit IgG antibody labeled with FITC (Zymed Laboratories, CA, USA). For counterstaining, cells were incubated for $20 \mathrm{~min}$ at $37^{\circ} \mathrm{C}$ with $2 \mu \mathrm{g} / \mathrm{ml}$ propidium iodide, PI (Sigma, MO, USA) and $20 \mu \mathrm{g} / \mathrm{ml}$ RNase A (Amresco Inc, OH, USA) in PBS. After washing, coverslips were mounted on microscope slides with VectaShield (Vector Laboratories Inc, CA, USA) and analyzed in a lasser scanning microscope (TCP-SP2, Leica, Heidelberg Germany) using $63 \times$ and $100 \times$ oil-immersion plan apochromat objectives (NA 1.32 and 1.4, respectively). The colocalization of FITC and PI staining was analyzed on single optical sections obtained for two channels throughout the $Z$ axis of the sample.

\section{Isolation of Cell Extracts}

Total, cytosolic, nuclear and nuclear matrix extracts were obtained as previously described $[19,26]$.

\section{Western Blot Analysis}

Equal amounts of protein extracts $(60 \mu \mathrm{g})$ were mixed with Tris-glycine SDS sample buffer and proteins were denatured by boiling $3 \mathrm{~min}$. Lysates were then separated by $10 \%$ SDS-PAGE, and electrotransferred to nitrocellulose membranes. Membranes were incubated for $1 \mathrm{~h}$ in TBS-T (150 mM NaCl, 10 mM Tris-HCl, pH 8, 0.05\% Tween 20) containing $6 \%$ low fat dried-milk and then incubated overnight with the corresponding primary antibody. After three washes with TBS-T, membranes were incubated with the appropriate secondary horseradish peroxidase-conjugated antibody (Amersham-Pharmacia, GE Healthcare, Buckinghamshire, UK) and developed using the ECL Western blotting analysis system (Amersham-Pharmacia, GE Healthcare, Buckinghamshire, UK).

\section{Immunoprecipitation}

Nuclear extracts in a final volume of $250 \mu$ were incubated for $1 \mathrm{~h}$ at $4^{\circ} \mathrm{C}$ with $5 \mu \mathrm{g}$ of DpC20 or CUT antibodies, previously bound to protein G-agarose (Invitrogen, Carlsbab, CA, USA). As negative control, parallel incubations with protein G-agarose and a non-related goat antibody 
bound to protein G-agarose were performed. The immune complexes were precipitated by centrifuging for $2 \mathrm{~min}$ at 2,500 rpm and washed twice in RIPA buffer $(160 \mathrm{mM}$ $\mathrm{NaCl}, 10 \mathrm{mM}$ Tris- $\mathrm{HCl}$ (pH 7.5), $1 \mathrm{mM}$ EDTA, $1 \mathrm{mM}$ EGTA, $20 \mathrm{mM}$ Na3MoO4, $20 \mathrm{mM} \mathrm{NaF}, 2 \mathrm{mM} \mathrm{NaVO} 4$, $1 \mathrm{mM}$ PMSF) containing complete protease inhibitor mixture (Roche Applied Science, IN, USA). Precipitated proteins were separated by SDS-PAGE and analyzed by Western blotting.

\section{Results}

Subcellular Localization of Dp71d and the DAPs in the PC12 and Antisense-Dp71 Cells

The presence of Dp71d and the dystrophin-associated proteins (DAPs) has been observed in the nuclei of HeLa and $\mathrm{C} 2 \mathrm{C} 12$ cells [26, 27]. Therefore, we decided to ascertain whether these proteins display nuclear localization in the PC12 neuronal cells. Furthermore, since Dp71 is a key component of the dystrophin-associated protein complex (DAPC) in different tissues and cultured cells lines [9, 28, 29], the influence of Dp71d on the expression and subcellular localization of DAPs was also evaluated in these cells. To accomplish this, PC12 cells stably-transfected with a plasmid that expresses a Dp71 antisense RNA (antisense-Dp71 cells) or with an empty vector (control cells) were isolated, as previously described [17]. The subcellular localization of Dp71 and DAPs was analyzed by immunofluorescence and confocal microscopy in the control and antisense-Dp71 cells, using antibodies directed specifically to Dp71d and each DAP ( $\beta$-dystroglycan, $\alpha / \beta$-dystrobrevin, $\varepsilon$-sarcoglycan, and $\alpha 1$-syntrophin) (Fig. 1). Prior to the selection of $\varepsilon$-sarcoglycan, it was demonstrated that PC12 control cells express $\alpha$-, $\beta$ - and $\delta$-sarcoglycan as
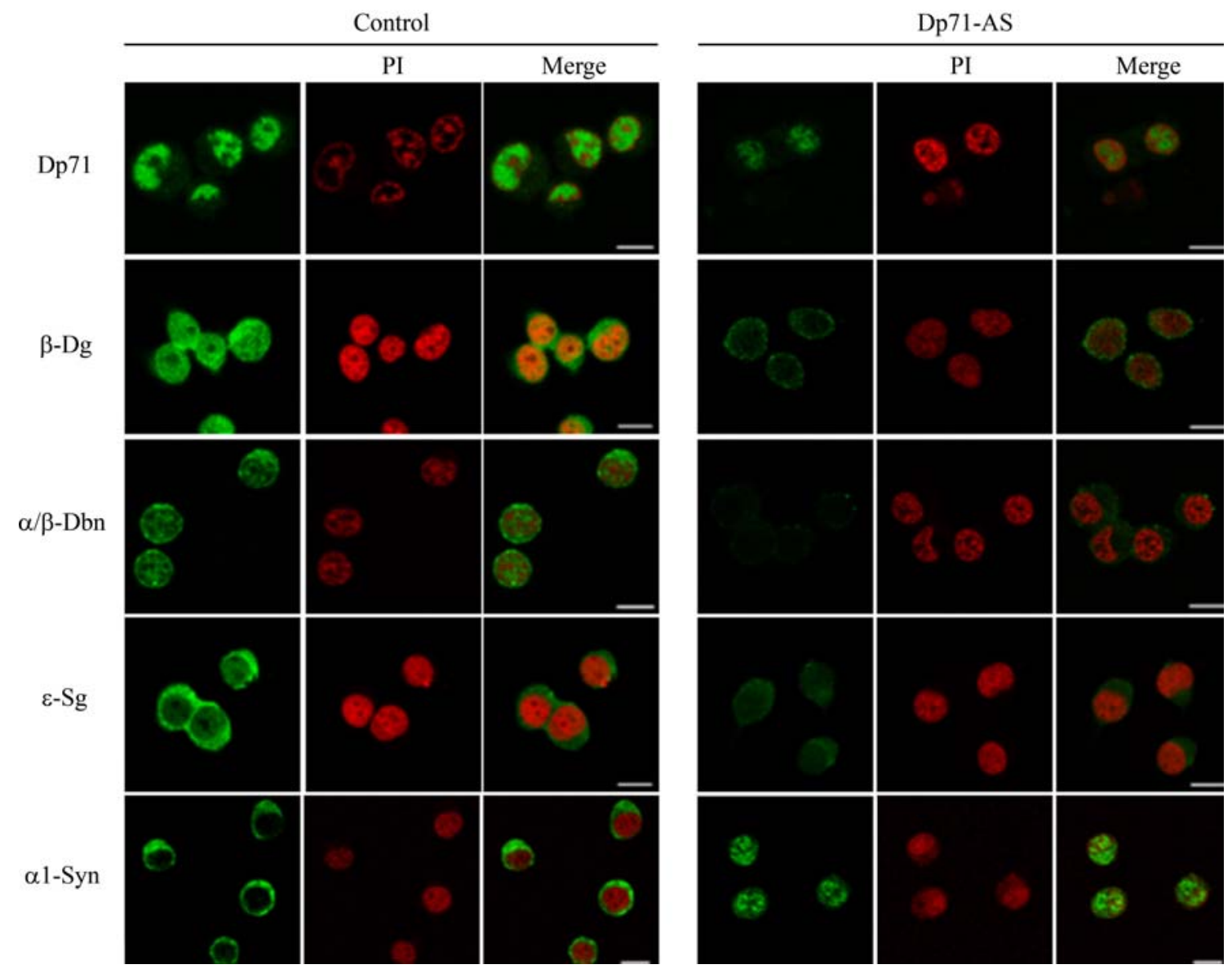

Fig. 1 Subcellular localization of Dp71d and DAPs in the control and antisense-Dp71 cells (Dp71-AS). Cells were cultured on glass coverslips and immunostained with primary antibodies directed to Dp71, $\beta$-dystroglycan $(\beta$-Dg), $\alpha$-/ $\beta$-dystrobrevin $(\alpha / \beta$-Dbn) or $\alpha 1$-syntrophin ( $\alpha 1$-Syn), and the specific signal was developed using the appropriate fluorescein-conjugated secondary antibody (green color). Cells were counterstained with propidium iodide (PI; red color) to visualize nuclei. After double labeling, cell preparations were subjected to confocal microscopy analysis and single optical $Z$-sections were selected in each case to show the subcellular distribution of each protein. Merged images are shown on the right panels. Bar $=10 \mu \mathrm{m}$ (For interpretation of the references to color in this figure legend, the reader is referred to the online version of this article) 
well. Nevertheless, their nuclear levels were scarce or null (Supplemental Fig.), in contrast with the evident nuclear localization of $\varepsilon$-sarcoglycan (see Fig. 1). In the control cells, the immunostaining of Dp71d was predominantly nuclear but excluded from nucleoli, while that of $\beta$-dystroglycan exhibited a dotted pattern that accumulated evenly between cytoplasm and nucleus. On the other hand, the pan-specific antibody directed to $\alpha / \beta$-dystrobrevin produced a discontinuous labeling localized through the cytoplasm and the nucleus, while the anti- $\varepsilon$-sarcoglycan antibody generated a predominant cytoplasmic labeling with a comparatively less intense signal in the nucleus. Finally, the localization of $\alpha 1$-syntrophin was almost entirely restricted to the cytoplasm of control cells. Similar results were obtained for the wild type PC12 cells (data not shown). As expected, the nuclear immunostaining of Dp71d was markedly reduced in the antisense-Dp71 cells. Interestingly, a drastic decrease in the immunolabeling of $\beta$-dystroglycan, $\alpha$-/ $\beta$-dystrobrevin and $\varepsilon$-sarcoglycan was observed in both the cytoplasm and nucleus of the antisense-Dp71 cells, compared with the control cells. On the other hand, the immunostaining of $\alpha 1$-syntrophin exhibited a slight increase in the antisense-Dp71 cells and its primary distribution changed from the cytoplasm to the nucleus.

\section{Dp71d is Critical for Stability of the DAPs in the PC12 Cells}

To corroborate the immunofluorescence results, with biochemical evidence, control and antisense-Dp71 cells were fractionated into total, cytoplasmic, nuclear and nuclear matrix extracts and further analyzed by Western blotting (Fig. 2), using antibodies against Dp71 and each DAP. The nuclear/cytoplasmic protein actin was used as loading control. To confirm the fractionation procedure fidelity, calnexin (an endoplasmic reticulum protein) and lamin A/C (a nuclear matrix-associated protein) were employed as cytoplasmic and nuclear marker, respectively (data not shown).

In the control cells, the immunoreactive band of Dp71d $(71 \mathrm{kDa})$ was recovered with similar intensity in the cytoplasmic and nuclear extracts and with a relative lower intensity in the nuclear matrix fraction. The $\beta$-dystroglycan protein band (43 $\mathrm{kDa}$ ) found mainly in the cytoplasm, was barely observed in the nucleus, while two retarded bands that migrate above the $50 \mathrm{kDa}$ marker were clearly observed in the nuclear and nuclear matrix fractions. The latter protein bands may correspond to the $65 \mathrm{kDa}$ isoform of $\beta$-dystroglycan previously reported in the brain [30] or alternatively might concur with hypoglycosylated moieties of the dystroglycan precursor. The immunoreactive band corresponding to $\beta$-dystrobrevin $(60 \mathrm{kDa})$ was distributed evenly in all of the cell fractions, while that of

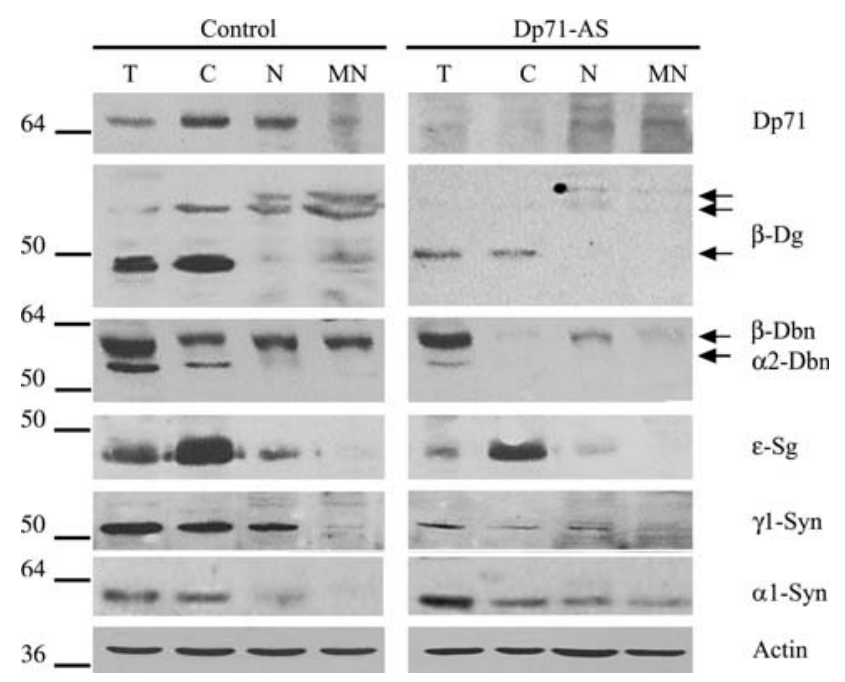

Fig. 2 Decreased levels of the DAPs in the different cell fraction of the antisense-Dp71 cells (Dp71-AS). Total (T), cytoplasmic (C), nuclear $(\mathrm{N})$ and nuclear matrix $(\mathrm{NM})$ extracts were isolated from the control and antisense-Dp71 cells. Equal amount of each protein extract $(60 \mu \mathrm{g})$ was resolved by SDS-PAGE and subjected to immunoblot analysis using antibodies directed to Dp71, $\beta$-dystroglycan $(\beta$-Dg), $\alpha$-dystrobrevin ( $\alpha$-Dbn), $\varepsilon$-sarcoglycan $(\varepsilon-\mathrm{Sg}), \alpha 1$-syntrophin $(\alpha 1$-Syn $)$ or $\gamma 1$-syntrophin $(\gamma 1$-Syn). As loading control, membranes were stripped and reproved with an anti-actin antibody. Position of protein markers is shown on the left. Arrows at the right denote the migration of the expected protein bands

$\alpha 2$-dystrobrevin $(55 \mathrm{kDa})$ was restricted to the cytoplasm. The $\varepsilon$-sarcoglycan $(43.5 \mathrm{kDa})$ was recovered in the cytoplasmic and nuclear extracts, being higher its concentration in the former fraction. Finally, $\alpha 1$-syntrophin (58 kDa) was prominent in the cytosolic fraction and practically absent in the nuclear extract, while $\gamma 1$-syntrophin $(50 \mathrm{kDa})$ distributed evenly between the cytoplasm and the nucleus. Similar data were obtained when the wild type PC12 cells were analyzed (data not shown). In the antisense-Dp71 cells, Dp71d was absent in the cytoplasm and barely detected in the nuclear and nuclear matrix extracts, confirming the efficacy of the antisense treatment. Noteworthy, with the exception of $\alpha 1$-syntrophin, a marked reduction in the levels of all of the DAPs tested was observed in the mutant cells, compared with either the wild type PC12 cells or the control cells. The $43 \mathrm{kDa} \beta$-dystroglycan decreased in the cytoplasm, while the isoform of $65 \mathrm{kDa}$ was undetectable in the nuclear and nuclear matrix extracts. Likewise, the protein expression of $\beta$-dystrobrevin decreased in all of the cell fractions, while that of $\alpha 2$-dystrobrevin decreased in the total extract and disappeared from the cytoplasmic fraction. Furthermore, the $\varepsilon$-sarcoglycan immunoreactive band displayed a slight reduction in the cytoplasm and the nucleus, whereas that of $\gamma 1$-syntrophin was drastically reduced in the same cell fractions. In contrast with the rest of the DAPs, the protein levels of $\alpha 1$-syntrophin increased in the nucleus and nuclear matrix extracts of the antisense-Dp71 
cells. Overall, the immunofluorescence and immunoblotting results established the presence of DAPs in the nucleus of PC12 cells and demonstrated the influence of Dp71 in the stability of the DAPs in both the cytoplasm and the nucleus.

Dp71d and the DAPs Form a Complex in the Nucleus of the PC12 Cells

The effect of the depletion of Dp71d on stability of the DAPs observed in the nucleus of the antisense-Dp71 cells implies that Dp71d interacts with these proteins within this organelle to assemble a dystrophin-associated protein complex (DAPC). To test this hypothesis, nuclear extracts from wild type PC12 cells were immunoprecipitated with the anti-Dp71 antibody DpC20 and the immunoprecipitated proteins were analyzed by Western blotting with antibodies directed to specific DAPs. In addition, the potential association of Dp71d with the nuclear actin was also evaluated. Figure 3 shows that Dp71d precipitated together with $\beta$-dystroglycan (43 and $65 \mathrm{kDa}$ isoforms), $\beta$-dystrobrevin, $\alpha 1$-syntrophin and actin. On contrary, we failed to immunoprecipitate $\gamma 1$-syntrophin with Dp71d (data not shown). None of these proteins was found when an irrelevant antibody (IgG0) was used for immunoprecipitation, establishing the specificity of the assay. These results demonstrated that Dp71 and the DAPs organize indeed a DAPC in the nucleus of the PC12 cells.

Utrophin Up71 Organizes a DAPC in the PC12 Cells but did not Compensate for Dp71d Deficiency in the Nucleus of Antisense-Dp71 cells

Utrophin gene, the autosomal homologue of the DMD gene, generates various protein products that are analogous to the dystrophin isoforms [31-33]. Then, the expression pattern of the utrophin gene was analyzed in the control and the antisense-Dp71 cells by immunoblotting using the anti-utrophin antibody CUT. This antibody is raised against the last 6 amino acids of the C-terminal domain of the fulllength utrophin (Up400) and recognizes all the utrophins $[21,22]$. Four different immunoreactive bands were revealed in both the control and the antisense-Dp71 cell extracts; based on their relative electrophoretic mobility these bands must correspond to Up400, Up140, Up113 and Up71 (Fig. 4a). To analyze whether utrophins assemble a DAPC in the PC12 cells, immunoprecipitation assays were carried out on total protein extracts from wild type PC12 cells, using the anti-utrophin antibody CUT. Immunoprecipitated proteins were analyzed by Western blotting using antibodies directed to specific DAPs. Figure $4 \mathrm{~b}$ shows that Up71 was immunoprecipitated specifically by the

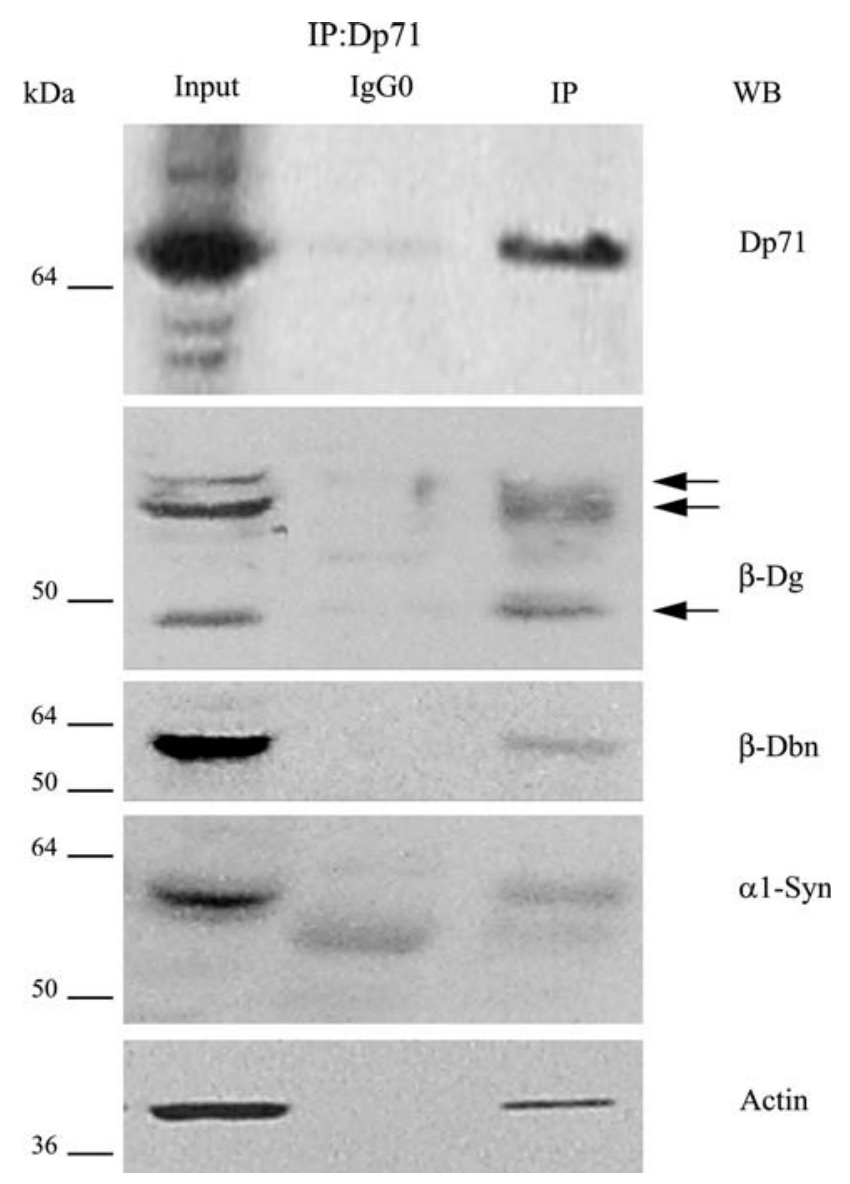

Fig. 3 Dp71d associates with the DAPs to form a protein complex in the nucleus of PC12 cells. Nuclear extracts from PC12 cells were immunoprecipitated with the anti-Dp71 antibody DpC20 and the immunoprecipitated proteins were analyzed by Western blotting with antibodies against Dp71, $\beta$-dystroglycan $(\beta$-Dg), $\beta$-dystrobrevin ( $\beta$-Dbn), $\alpha 1$-syntrophin ( $\alpha 1$-Syn) and actin. IP, immunoprecipitation; WB, immunoblotting. IgG0, irrelevant IgG used as negative control. Input, $10 \%$ of the nuclear extract was subjected to immunoblotting without previous immunoprecipitation. Arrows at the right denote the migration of the expected protein bands

anti-utrophin antibody CUT and that $\alpha 1$-syntrophin, $\beta$-dystroglycan, $\beta$-dystrobrevin and actin were pull-down together with this utrophin.

It is well known that Up400 compensates for dystrophin deficiency in muscle tissue [34]. Therefore, it is likely that Up71, the counterpart of Dp71, might compensate for depleted levels of Dp71d isoform in the antisense-Dp71 cells. To test this idea, cell fractions from control and mutant cells were analyzed by immunoblotting for detecting Up71 and actin (loading control). Figure 4c shows that Up71 protein levels were increased in the total and cytoplasmic extracts of antisense-Dp71 cells, compared with control cells: However, Up71 was barely detected in the nucleus of control cells and such a reduced nuclear expression showed no increase in the mutant cells. 

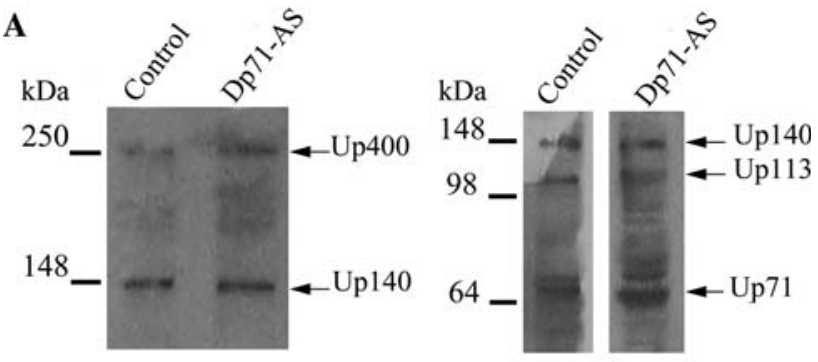

B

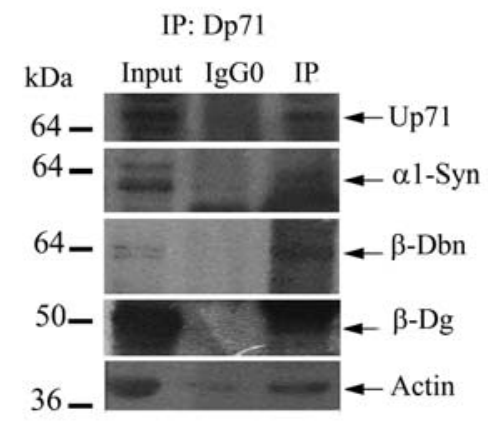

C

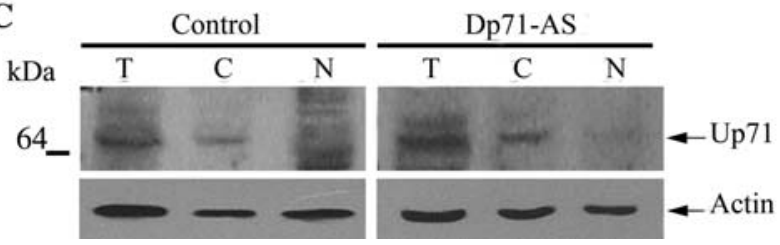

Fig. 4 Expression of utrophin gene products in the PC12 cells. a Total protein extracts from the control and the antisense-Dp71 cell cultures were analyzed by SDS-PAGE (polyacrilamide at 5 or $10 \%$ was used for the left and right gels, respectively) and subjected to immunoblotting analysis, using the anti-utrophin antibody CUT. The expected utrophin isoforms are denoted by arrows. b Total protein extracts from the control PC12 cells were immunoprecipitated with the anti-utrophin antibody CUT and the precipitated proteins were analyzed by immunoblotting with antibodies raised against utrophin (CUT), $\alpha 1$-syntrophin ( $\alpha 1$-Syn), $\beta$-dystrobrevin $(\beta$-Dbn), $\beta$-dystroglycan $(\beta$-Dg) and actin. IP, immunoprecipitation; WB, immunoblotting. $\operatorname{IgG} 0$, irrelevant $\operatorname{IgG}$ used as negative control. Input, $10 \%$ of the total protein extract subjected to immunoblotting without previous immunoprecipitation. Arrows at the right denote the migration of the expected protein bands. c Total (T), cytoplasmic (C), and nuclear $(\mathrm{N})$ protein extracts isolated from control and Dp71-antisense cells were resolved by SDS-PAGE and subjected to immunoblotting analysis using the anti-utrophin antibody. Membranes were stripped and reproved with an anti-actin antibody (loading control). Arrow on the right denotes the migration of Up71 protein band. Migration of the protein markers is shown on the left of each panel

\section{Discussion}

We [17, 19], and others [18, 35, 36], have adopted the PC12 cell line as in vitro neuronal model for studying the function of Dp71. These cells express two Dp71 isoforms called Dp71d and Dp71f, which display different subcellular localization each other [18]. Dp71d is distributed predominantly to the nucleus, while Dp71f localizes exclusively at cytoplasm. To ascertain the role of each Dp71 isoform, we have previously isolated by antisense treatment against the Dp71 mRNA, a PC12-derivative clone with deficient expression of Dp71 (antisense-Dp71 cells). Interestingly, antisense-Dp71 cells exhibit deficient adhesion to substrate and impaired neurite outgrowth in response to NGF induction [17, 37]. Previously, we demonstrated that the Dp71f variant modulates the adhesion of PC12 cells by interacting with the $\beta 1$-integrin adhesion protein complex and by this way conferring stability to this complex. Furthermore, it has been reported that Dp71f associates with $\beta$-dystroglycan, $\alpha 1$-syntrophin and $\beta$-dystrobrevin in the cytoplasm of the PC12 cells to constitute a dystrophin-associated protein complex (DAPC) [36]. These authors propose that the cytoplasmic DAPC is involved in the cytoskeleton network modeling that occurs during the NGF-induced neuronal differentiation of PC12 cells. On the other hand, it seems that the Dp71d isoform is involved somehow in the NGF-induced neurite outgrowth of these cells; because, its over-expression enhances the extension of neurites [17].

In this study, we show the presence of different dystrophin- associated proteins (DAPs) in the nucleus of PC12 cells, namely $\beta$-dystroglycan, $\beta$-dystrobrevin, $\alpha 1$-syntrophin, $\gamma 1$-syntrophin and $\varepsilon$-sarcoglycan, and demonstrated that $\mathrm{Dp} 71 \mathrm{~d}$ interacts with $\beta$-dystroglycan, $\beta$-dystrobrevin and $\alpha 1$-syntrophin within this organelle to assemble a nuclear DAPC. By the analysis of the antisense-Dp71 cells, we revealed that depletion of Dp71 caused a drastic reduction in the levels of $\beta$-dystroglycan, $\beta$-dystrobrevin, $\varepsilon$-sarcoglycan and $\gamma 1$-syntrophin in both the cytoplasm and the nucleus, compared with either the wild type PC12 cells or the control cells (stably-transfected with the empty vector). On contrary, the nuclear levels of $\alpha 1$-syntrophin increased in the nucleus of the mutant cells, suggesting that this protein might enter the nucleus to compensate for the lack of $\gamma 1$-syntrophin. Taking into account the predominant subcellular localization of each Dp71 isoform in the PC12 cells, our results strongly suggest that Dp71f and Dp71d are critical for the stability of the DAPs in the cytoplasm and nucleus, respectively. These findings are consistent with previous reports that highlighted the importance of Dp71 on the stability of DAPs. Dp71 is able to restore the normal levels and localization of DAPs in the sarcolemma of the $m d x$ mice [28]. In concordance, the mutant mice $\mathrm{mdx}^{3 \mathrm{cv}}$, which do not express Dp71, have reduced levels of DAPs in their brain [29]. However, it is worth to mention that this study is the first to demonstrate the critical role of Dp71 on the organization and/or stability of the DAPC in a different cell compartment, the nucleus.

We showed that different utrophin gene products are expressed in the PC12 cells (Up400, Up140, Up113, and Up71); furthermore, we demonstrated that Up71 organized 
an alternative DAPC in these cells, which includes at least $\alpha 1$-syntrophin, $\beta$-dystroglycan, $\beta$-dystrobrevin and actin. Since utrophins are functional homologous of dystrophin isoforms [31-33], we hypothesized that Up71, the counterpart of Dp71, might compensate for Dp71d deficiency in the nucleus of the antisense-Dp71 cells. Although Up71 total protein levels exhibited an increase in the antisenseDp71 cells, compared with the control cells, this protein was barely detected in the nucleus of both cell cultures. Hence it is unlikely that Up71 could replace the nuclear function of Dp71d.

The nuclear DAPC is not cell-type specific, we have characterized similar complexes in the nucleus of HeLa [26] and C2C12 cells [27], which suggests an important unknown role for this complex in nuclear activities. We found that Dp71d as well as $\beta$-dystroglycan and $\beta$-dystrobrevin are associated with the nuclear matrix, a permanent network of core filaments underlying ticker fibers which is proposed to be the platform for numerous nuclear processes, including RNA processing, transcription regulation and DNA repair. Therefore, we favored the idea that the nuclear DAPC has a structural role in the PC12 cell nuclei and by this way modulates nuclear matrix-associated processes. Consistent with this, we have previously revealed that Dp71d and some DAPs ( $\beta$-dystroglycan and $\beta$-dystrobrevin) interact with the nuclear matrix proteins actin and lamin B1 in the HeLa cells [26]. In this scenery, it is tempting to speculate that depletion of Dp71 and consequently of the DAPs has negative consequences for the nuclear function of the antisense-Dp71 cells. Currently, different cellular and molecular strategies are being approached in our laboratory to identify potential alterations in the nucleus of the mutant cells.

Acknowledgments We are grateful to Dr. Manuel Hernández (Department of Cellular Biology, CINVESTAV-IPN, Mexico City) for supplying anti-actin monoclonal antibody and Dr. Derek J. Blake (Department of Human Anatomy and Genetics, University of Oxford, Oxford, UK) for providing 2166 and $\beta$-CTFP antibodies. We also thank to Pablo Gómez and Victor Tapia for technical assistance. This work was supported by CONACYT-México, Grant No. 54858, (to BC). CONACYT also granted scholarships for PhD students (183964, 206575 control numbers).

\section{References}

1. Emery AE (1987) X-linked muscular dystrophy with early contractures and cardiomyopathy (Emery-Dreifuss type). Clin Genet 32:360-367

2. Koenig M, Hoffman EP, Bertelson CJ et al (1987) Complete cloning of the Duchenne muscular dystrophy (DMD) cDNA and preliminary genomic organization of the DMD gene in normal and affected individuals. Cell 50:509-517

3. Mehler MF (2000) Brain dystrophin, neurogenetics and mental retardation. Brain Res Brain Res Rev 32:277-307
4. Yoshida M, Ozawa E (1990) Glycoprotein complex anchoring dystrophin to sarcolemma. J Biochem 108:748-752

5. Byers TJ, Lidov HG, Kunkel LM (1993) An alternative dystrophin transcript specific to peripheral nerve. Nat Genet 4:77-81

6. D'Souza VN, Nguyen TM, Morris GE et al (1995) A novel dystrophin isoform is required for normal retinal electrophysiology. Hum Mol Gene 4:837-842

7. Lederfein D, Yaffe D, Nudel U (1993) A housekeeping type promoter, located in the $3^{\prime}$ region of the Duchenne muscular dystrophy gene, controls the expression of Dp71, a major product of the gene. Hum Mol Genet 2:1883-1888

8. Lidov HG, Kunkel LM (1997) Dp140: alternatively spliced isoforms in brain and kidney. Genomics 45:132-139

9. Claudepierre T, Dalloz C, Mornet D et al (2000) Characterization of the intermolecular associations of the dystrophin-associated glycoprotein complex in retinal Muller glial cells. J Cell Sci 113(Pt 19):3409-3417

10. Hugnot JP, Gilgenkrantz H, Vincent N et al (1992) Distal transcript of the dystrophin gene initiated from an alternative first exon and encoding a $75-\mathrm{kDa}$ protein widely distributed in nonmuscle tissues. Proc Natl Acad Sci USA 89:7506-7510

11. Lederfein D, Levy Z, Augier N et al (1992) A 71-kilodalton protein is a major product of the Duchenne muscular dystrophy gene in brain and other nonmuscle tissues. Proc Natl Acad Sci USA 89:5346-5350

12. Moizard MP, Billard C, Toutain A et al (1998) Are Dp71 and Dp140 brain dystrophin isoforms related to cognitive impairment in Duchenne muscular dystrophy? Am J Med Genet 80:32-41

13. Moizard MP, Toutain A, Fournier D et al (2000) Severe cognitive impairment in DMD: obvious clinical indication for Dp71 isoform point mutation screening. Eur J Hum Genet 8:552-556

14. Jung D, Yang B, Meyer J et al (1995) Identification and characterization of the dystrophin anchoring site on beta-dystroglycan. J Biol Chem 270:27305-27310

15. Sarig R, Mezger-Lallemand V, Gitelman I et al (1999) Targeted inactivation of Dp71, the major non-muscle product of the DMD gene: differential activity of the Dp71 promoter during development. Hum Mol Genet 8:1-10

16. Greene LA, Tischler AS (1976) Establishment of a noradrenergic clonal line of rat adrenal pheochromocytoma cells which respond to nerve growth factor. Proc Natl Acad Sci USA 73:2424-2428

17. Acosta R, Montañez C, Fuentes-Mera L et al (2004) Dystrophin Dp71 is required for neurite outgrowth in PC12 cells. Exp Cell Res 296:265-275

18. Marquez FG, Cisneros B, Garcia F et al (2003) Differential expression and subcellular distribution of dystrophin Dp71 isoforms during differentiation process. Neuroscience 118:957-966

19. Rodríguez-Muñoz R, Villarreal-Silva M, Gonzalez-Ramirez R et al (2008) Neuronal differentiation modulates the dystrophin Dp71d binding to the nuclear matrix. Biochem Biophys Res Commun 375:303-307

20. Rivier F, Robert A, Hugon G et al (1999) Dystrophin and utrophin complexed with different associated proteins in cardiac Purkinje fibres. Histochem J 31:425-432

21. Claudepierre T, Mornet D, Pannicke T et al (2000) Expression of Dp71 in Muller glial cells: a comparison with utrophin- and dystrophin-associated proteins. Invest Ophthalmol Vis Sci 41:294-304

22. Fabbrizio E, Leger J, Anoal M et al (1993) Monoclonal antibodies targeted against the C-terminal domain of dystrophin or utrophin. FEBS Lett 322:10-14

23. Blake DJ, Nawrotzki R, Loh NY et al (1998) Beta-dystrobrevin, a member of the dystrophin-related protein family. Proc Natl Acad Sci USA 95:241-246

24. Blake DJ, Hawkes R, Benson MA et al (1999) Different dystrophin-like complexes are expressed in neurons and glia. J Cell Biol 147:645-658 
25. Díaz-Barriga CL, Yáñez L, Hernández JM et al (1989) Interaction of cadmium with actin microfilaments in vitro. Toxic in vitro 3:277-284

26. Fuentes-Mera L, Rodríguez-Muñoz R, Gonzalez-Ramirez R et al (2006) Characterization of a novel Dp71 dystrophin-associated protein complex (DAPC) present in the nucleus of HeLa cells: members of the nuclear DAPC associate with the nuclear matrix. Exp Cell Res 312:3023-3035

27. Gonzalez-Ramirez R, Morales-Lazaro SL, Tapia-Ramirez V et al (2008) Nuclear and nuclear envelope localization of dystrophin Dp71 and dystrophin-associated proteins (DAPs) in the $\mathrm{C} 2 \mathrm{C} 12$ muscle cells: DAPs nuclear localization is modulated during myogenesis. J Cell Biochem 105:735-745

28. Cox GA, Sunada Y, Campbell KP et al (1994) Dp71 can restore the dystrophin-associated glycoprotein complex in muscle but fails to prevent dystrophy. Nat Genet 8:333-339

29. Greenberg DS, Schatz Y, Levy Z et al (1996) Reduced levels of dystrophin associated proteins in the brains of mice deficient for Dp71. Hum Mol Genet 5:1299-1303

30. Royuela M, Chazalette D, Hugon G et al (2003) Formation of multiple complexes between beta-dystroglycan and dystrophin family products. J Muscle Res Cell Motil 24:387-397
31. Jimenez-Mallebrera C, Davies K, Putt W et al (2003) A study of short utrophin isoforms in mice deficient for full-length utrophin. Mamm Genome 14:47-60

32. Lumeng CN, Phelps SF, Rafael JA et al (1999) Characterization of dystrophin and utrophin diversity in the mouse. Hum Mol Genet 8:593-599

33. Wilson J, Putt W, Jimenez C et al (1999) Up71 and up140, two novel transcripts of utrophin that are homologues of short forms of dystrophin. Hum Mol Genet 8:1271-1278

34. Grady RM, Teng H, Nichol MC et al (1997) Skeletal and cardiac myopathies in mice lacking utrophin and dystrophin: a model for Duchenne muscular dystrophy. Cell 90:729-738

35. Cisneros B, Rendon A, Genty V et al (1996) Expression of dystrophin Dp71 during PC12 cell differentiation. Neurosci Lett 213:107-110

36. Romo-Yáñez J, Ceja V, Ilarraza-Lomeli R et al (2007) Dp71ab/ DAPs complex composition changes during the differentiation process in PC12 cells. J Cell Biochem 102:82-97

37. Enriquez-Aragon JA, Cerna-Cortes J, Bermudez de Leon $\mathrm{M}$ et al (2005) Dystrophin Dp71 in PC12 cell adhesion. Neuroreport $16: 235-238$ 